\title{
Measuring Higher Dimensional "Qudits" for Computation
}

\author{
With a technique called self-guided tomography, researchers accurately \\ measure the states of qudits-quantum systems like qubits but with more \\ than two dimensions.
}

By Erika K. Carlson

$I$ n classical computing, a bit (binary digit) has two dimensions by definition. Quantum computers employ qubits, the classical bit's quantum equivalent, but could also use qudits, quantum systems with $d$ potential states or dimensions. Markus Rambach of the University of Queensland in Australia and colleagues have now brought such an approach a step closer to reality by showing that a particular technique for measuring quantum states works for higher-dimensional systems than previously tested [1].

To use qudits to their full potential, researchers must be able to create them, control them, and measure their states. States of qudits are measured using a class of techniques called quantum state tomography, but the measurements grow more challenging as the number of dimensions in a system increases. One approach, called self-guided tomography, might allow high accuracy and precision with fewer measurements compared with other quantum tomographic techniques. However,

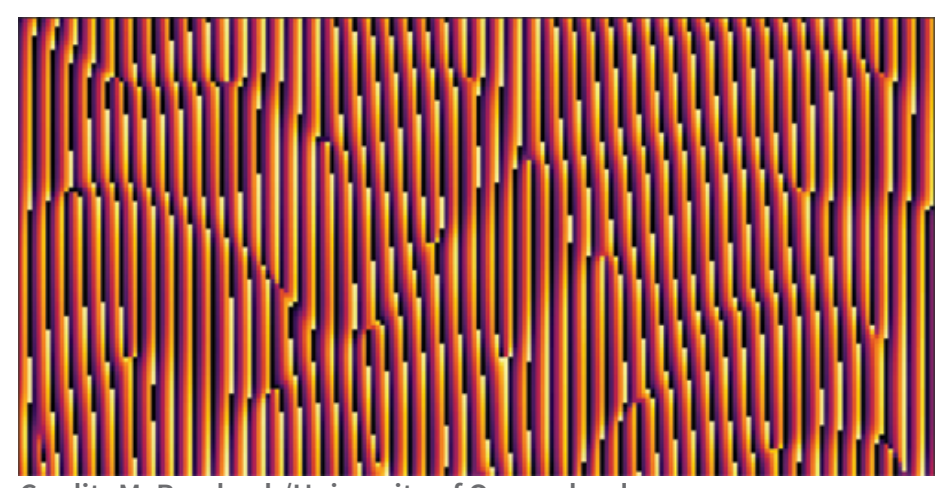

Credit: M. Rambach/University of Queensland self-guided tomography has so far only been tested on low-dimensional systems, such as a system of two qubits, which has a total number of dimensions $d=4$.

Rambach and colleagues tested self-guided tomography on pure-state qudits-states that can be written as single vectors in a complex Hilbert space-with 3, 5, and 20 dimensions. They found that the technique is effective for such high-dimensional systems, achieving measurement fidelities of over $99 \%$ for all three cases. Though self-guided tomography was originally proposed for measuring pure states, the researchers extended the method to deal with mixed states, demonstrating measurement fidelities of about $95 \%$ or higher for mixed-state qudits of three dimensions. Such high-fidelity measurements will likely be necessary to read the mixed-state outputs expected from quantum computers based on high-dimensional qudits

Erika K. Carlson is a Corresponding Editor for Physics based in New York City.

\section{REFERENCES}

1. M. Rambach et al., "Robust and efficient high-dimensional quantum state tomography," Phys. Rev. Lett. 126, 100402 (2021). 